\title{
Einleitung: Schulbücher und Lektüren in der vormodernen Unterrichtspraxis
}

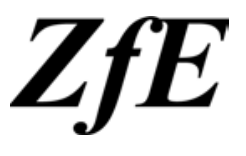

\author{
Stephanie Hellekamps $\cdot$ Jean-Luc Le Cam $\cdot$ Anne Conrad
}

\section{Introduction: schoolbooks and reading in early modern lessons}

Lesen zu können ist nicht nur eine wesentliche Voraussetzung für den Erwerb von Kenntnissen in nahezu allen Schulfächern sowie für Ausbildung und Studium, sondern für die Teilhabe am gesellschaftlichen Leben in der Moderne überhaupt. In den PISA-Untersuchungen kommt der Lesekompetenz daher eine Schlüsselstellung unter den getesteten Kompetenzen zu. Sie gilt als ,universelles Kulturwerkzeug“, da ohne Lesekompetenz eine selbstbestimmte und damit befriedigende Lebensführung in der Moderne nicht möglich ist (Klieme et al. 2010, S. 24). PISA geht bei der Erfassung von Leseleistungen von einem weiten Kompetenzbegriff aus: Da das Lesen für die kulturelle Teilhabe während des gesamten Lebenslaufs von entscheidender Bedeutung bleibt, intendiert schulischer Unterricht nicht nur die Befähigung der Heranwachsenden dazu, Texte unterschiedlicher Schwierigkeitsgrade zu verstehen und zu beurteilen. Vielmehr geht es auch um die Ausbildung einer Haltung, die das Lesen als kulturelle Praktik und ,die aktive Teilhabe an der Gemeinschaft der Lesenden“" schätzt (ebd., S. 74). Inzwischen besitzen wir dank internationaler Forscherteams und komplexer Erhebungsmethoden differenziertes Wissen darüber, auf welchen Kompetenzniveaus Jugendliche der verschiedenen OECDund anderer Staaten lesen können, welche Lesestrategien sie praktizieren, wie groß ihre Lesemotivation ist etc.

(C) Springer Fachmedien Wiesbaden 2012

\author{
S. Hellekamps $(\bowtie)$ \\ Institut für Erziehungswissenschaft, Westf. Wilhelms-Universität Münster, \\ Bispinghof 5/8, 48143 Münster, Deutschland \\ E-Mail: hellekam@uni-muenster.de \\ J.-L. Le Cam \\ Université de Bretagne Occidentale, Brest 35 rue Léo Lagrange, \\ 29000 Quimper, Frankreich \\ E-Mail: Jean-Luc.LeCam@univ-brest.fr \\ A. Conrad \\ Institut für Katholische Theologie, Universität des Saarlandes, \\ Campus A 4.2,66123 Saarbrücken, Deutschland \\ E-Mail: a.conrad@mx.uni-saarland.de
}


Sehr viel geringer ist hingegen unsere Kenntnis der vormodernen Lesepraktiken, deren Erforschung ganz eigene Wege und Verfahren erfordert. Anlässlich seiner 13. Tagung, die im November 2011 am Zentrum für interdisziplinäre Forschung in Bielefeld (ZiF) stattfand, hat sich der „Arbeitskreis für die Vormoderne in der Erziehungsgeschichte“ (AVE) mit Fragen nach Inhalten und Methoden des Lesens und Lernens von Kindern und Jugendlichen in der Vormoderne befasst. Welche Texte und Autoren haben Kinder und Jugendliche im späten Mittelalter und in der frühen Neuzeit gelesen, und wie wurden ihre Lektüren von Lehrern, Lehrerinnen, Eltern und anderen Erziehern angeregt? Wie gestaltete sich die Normierung, Produktion und Verbreitung von Schulbüchern? An regionalen Beispielen aus Frankreich, den Niederlanden und dem Alten Reich wird in den aus der Tagung hervorgegangenen Beiträgen gezeigt, welche Schulbücher und anderen Lektüren das Lernen der Heranwachsenden bestimmt haben und welche Praktiken des Lesens im Unterricht vorherrschend waren. Die Untersuchungen beziehen sich auf die Zeit des Spätmittelalters bis zur ersten Hälfte des 18. Jahrhunderts und schließen Elementarbildung und Sekundarunterricht an Schulen, das häusliche Selbststudium und die Unterweisung im höfischen Kontext ein. Auch der Zusammenhang von Alphabetisierung und religiöser Bildung wird erörtert.

Unter ,Schulbüchern ' werden zum einen solche Texte verstanden, die von ihren Autoren explizit zu Unterrichtszwecken verfasst wurden, wie z.B. Grammatiken, Vokabularien und Katechismen, und die von Kindern und Jugendlichen in der Schule zum Lernen verwendet wurden. Zum anderen gehören dazu Texte, deren Beziehung zum Schulunterricht durch zeitgenössische Zeugnisse nachgewiesen ist, ohne dass die Verwendung im schulischen Kontext von den Autoren intendiert worden war. Beispiele für diese letztere Textsorte sind etwa die antiken und mittelalterlichen Auctores oder religiöse Schriften. ,Andere Lektüren ' umfassen solche Unterrichtstexte, die Kinder und Jugendliche in Gebrauchssituationen außerhalb der Schule zum Lernen genutzt haben.

$\mathrm{Um} \mathrm{zu}$ erforschen, wie gelesen und gelernt wurde, ist die Kombination verschiedener sowohl normativer als auch von Schülern benutzter Quellen und qualitativer und quantitativer Methoden unumgänglich. Das hängt mit der ungewöhnlich dürftigen Überlieferung zusammen. Schul- und Unterrichtsbücher wurden in besonderem Maße durch Gebrauch verbraucht und damit von weiterer Überlieferung ausgeschlossen (vgl. Henkel 1988, S. 7 f.). Die wenigen erhaltenen Exemplare sind zumeist solche, die frühzeitig gebunden und dadurch der Nutzung entzogen und konserviert wurden (vgl. ebd., S. 149). Ein neueres Beispiel für diese Nicht-Benutzung im Schulunterricht stellen die beiden von Hergenhahn gefundenen und publizierten Exemplare eines Dortmunder Rechenbuches aus dem 16. Jh. dar (Hergenhahn 2007). Damit aber fehlen ihnen gerade diejenigen Spuren des Gebrauchs, die Aufschluss über die Praktiken des Lesens und Lernens geben könnten. Wo sich hingegen bei erhaltenen Exemplaren von Lehrwerken Gebrauchsspuren in Form von Besitzvermerken, Datumsangaben und Glossen finden, sind mitunter Rückschlüsse auf die tatsächlichen Verwendungsweisen der Bücher im Unterricht möglich. Bernhard hat für die Rezeption von Boethius' Schriften zum Quadrivium im Schulunterricht aus der Unterscheidung von Typen der Glossierung gezeigt, wie auf spezifische Methoden des Unterrichts geschlossen werden kann (Bernhard 1996, S. 23). Die Untersuchung von Texten lateinischer Klassiker, die mit grammatikalischen Erläuterungen und Erklärungen für den Unterricht des französischen Dauphins herausgegeben wurden, gibt 
ebenfalls Aufschluss über den Gebrauch dieser Texte im Unterricht (vgl. Volpilhac-Auger 2000). Hinweise $\mathrm{zu}$ den wirklichen Lektürepraktiken können auch aus der Einteilung eines Inhalts auf einer gedruckten Buchseite gewonnen werden. Satz, Umbruch, Bilder, leere Seiten für Notizen etc. haben das Lesen mitbestimmt, die Sehweise des lesenden Schülers/der Schülerin dirigiert und dadurch ihre Rezeptionen mitgesteuert (vgl. Martin 2000).

Gleichwohl bleibt es schwierig, die Anwendungszusammenhänge der überlieferten Texte zu rekonstruieren (vgl. Blair 2008, S. 39 ff.). Schulpolitische Vorschriften, Schulordnungen und Schulprogramme bieten zwar weitere Anhaltspunkte. Doch bekanntlich besteht zwischen Norm und Praxis eine Kluft. So haben Bodemann und Dabrowski durch Vergleich des Überlieferungsbestandes von Handschriften der Ulmer Lateinschule mit den Schulordnungen und Lektionsplänen dieser Schule aus dem 15. Jh. festgestellt, dass die Unterrichtswirklichkeit vom Plan abwich. Im Elementarunterricht wurden neben den im Lektionsplan vorgegebenen beiden Standardgrammatiken auch mehrere Grammatiken verwendet, die weder in den Schulordnungen noch in den Lektionsplänen erwähnt wurden (Bodemann und Dabrowski 2000, S. 39). Eine Diskrepanz zwischen den normativen Vorgaben und dem wirklichen Unterricht haben auch die Studien von Albertini (1986), Caspard (1990) und Compère und Pralon-Julia (1992) nachgewiesen. Überdies lässt die Konzentration auf das schulische Lernen die außerschulischen Praktiken des Erwerbs von Wissen und Können außer Acht. Damit aber bleibt das Lernen der Mädchen, das oft im häuslichen Kontext stattfand, zumeist unberücksichtigt (vgl. Jacobi 2008, S. 273).

Um trotz der lückenhaften Überlieferung zu neuen Erkenntnissen über die vormodernen Lektürepraktiken zu kommen, werden in einigen unserer Beiträge überlieferte (Schul-) Bibliotheksbestände mit ihren Inventarverzeichnissen und Bücherlisten ausgewertet. Dies hat sich sowohl im Kontext der höfischen Unterweisung als auch in der Konventserziehung als fruchtbar erwiesen, wie die Beiträge von Pascale Mormiche, Dominique Picco und Anne Conrad zeigen. Ferner ergab die Untersuchung der Auflagenhöhe und des Verbreitungsgrades von Schulbüchern Hinweise auf ihre tatsächliche Nutzung. In diesem Zusammenhang wurde nach der Bedeutung örtlicher Offizinen für den Schulbuchdruck gefragt. Hinsichtlich der geographischen Verbreitung von Schulbüchern hatte bereits Puff für den deutschsprachigen Raum 1480-1560 eine weite Streuung festgestellt: „Im allgemeinen bedienten gedruckte Unterrichtstexte nicht nur einen städtischen oder territorialen Buchmarkt, sondern reichten darüber hinaus.“ (1996, S.259) Tatsächlich wird die Erforschung der technischen Herstellung, Auflagenhöhe und geographischen Verbreitung von Schulbüchern durch ihre geringe Beachtung in Nachlässen und durch die geringe Zahl überlieferter Inventare oder Rechnungen von Buchhändlern erschwert, wie schon Dainville (1947/1978) und Desgraves (1973) festgestellt haben. Die bisherigen Forschungsbefunde können nun insbesondere durch die wirtschaftsgeschichtlich akzentuierte Studie Emmanuelle Chaprons ergänzt werden. Chapron zufolge kann von einem einheitlichen Markt für Schulbücher, der durch Angebot und Nachfrage bestimmt worden wäre, nicht gesprochen werden, da nicht nur ökonomische Strategien, sondern auch administrative Verordnungen, Familienbeziehungen und Netzwerke der Händler Produktion und Distribution von Schulbüchern regulierten. Das Ineinander von obrigkeitlich festgesetzter Norm (z. B. hinsichtlich der zu verwendenden Schulbücher) und (kontrollierter) Praxis studiert Jean-Luc Le Cam am Beispiel der Schulpolitik im Herzogtum Braunschweig-Wolfenbüttel. Aufschluss 
über den Gebrauch von Lernmaterialien im Unterricht in der Spannung von Norm und Praxis geben (Schul-)Inspektionsberichte, die sich als weitere wichtige Quellen erwiesen.

Mit ihrem interdisziplinären Zugang erprobte die AVE-Tagung somit verschiedene Wege und Methoden, um die tatsächlichen Lektürepraktiken von Kindern und Jugendlichen in den Blick zu bringen. Der vorliegende Band präsentiert die Ergebnisse dieses Austausches, an dem sich Vertreterinnen und Vertreter der Geschichtswissenschaft, Erziehungswissenschaft, Theologie und Germanistik beteiligt haben. Die bereits erwähnte geographische Beschränkung gewährleistet die Vergleichbarkeit der Studien, die sozialhistorische, bildungs- und schulgeschichtliche Perspektiven verschränken und auch den konfessionellen Aspekt mit berücksichtigen. Wesentliche Impulse bezog die Diskussion aus der mediävistischen und frühneuzeitlichen Germanistik, die sich seit den späten 80er Jahren verstärkt der Erforschung des Verhältnisses von Mündlichkeit und Schriftlichkeit im Unterrichtsgeschehen vom 9. bis zum 17. Jh. zugewandt hat. Durch die Untersuchung von Schul-Handschriften wurde die Geschichte des Zusammenhangs von schriftlicher und mündlicher Kommunikation im Trivialunterricht des deutschen Reichs als Abfolge von Verschriftlichungsschüben eines wesentlich mündlichen Geschehens nachgewiesen (vgl. Baldzuhn 2009; Bodemann und Grubmüller 1992; Grubmüller 2000). Diesem Schwerpunkt war auch der Eröffnungsvortrag gewidmet, mit dem zugleich dieser Band beginnt, weil er in grundlegender Weise auf die methodischen Probleme verweist, die sich ergeben, wenn die Eigenart des vormodernen Lernens aufgehellt werden soll.

In seinem Beitrag Das Ritual des Textverstehens. Zu Konzeption und Gebrauch des spätmittelalterlichen Schulbuchs betont Michael Baldzuhn, dass sich vormoderner Unterricht für die bildungshistorische Forschung als ein nur schwach institutionalisiertes Geschehen mit allenfalls gering ausgeprägter organisatorischer und didaktisch-methodischer Selbstreflexion präsentiert. So ist oft nur schwer zu entscheiden, ob ein überliefertes Schulbuch ursprünglich für die Hand des Lehrers oder des Schülers oder beider gedacht war. Auch wo sich an den Büchern Nutzungsspuren in Form von Interlinearglossen oder Kommentaren zeigen, könne nicht unmittelbar auf die Unterrichtspraktiken geschlossen werden, denen sich diese Spuren angeblich verdanken. Denn auch die Kommentierung selbst sei entscheidend durch Vorlagen tradiert worden. Wie es gleichwohl möglich ist, zu einer Vorstellung des individuellen Unterrichts zu gelangen, veranschaulicht Baldzuhn am Arrangement von Text, Kommentar und Glossen der Disticha Catonis sowie der spätantiken Fabeln des Avian auf handgeschriebenen, später gedruckten Seiten. Indem er auch den medialen Aspekt des Buchs beleuchtet, verfolgt Baldzuhn, in welchem Maße Schüler und Lehrer in schriftlichen und mündlichen Praktiken am spätmittelalterlichen (Latein-)Unterricht beteiligt waren.

Den mit der Erfindung des Buchdrucks erweiterten Möglichkeiten des Schriftspracherwerbs ist der Beitrag Frühe Lese- und Schreiblernbücher des 16. Jahrhunderts. Zu Valentin Ickelsamers Die rechte weis, aufs kürzist lesen zu lernen (1527) und Teütsche Grammatica (1532) von Hans Rudolf Velten gewidmet. Die beiden von Velten untersuchten Texte repräsentieren den Typus der frühneuzeitlichen Anleitungsschrift, die das „Selbstlernen“ betonte und sich als vielseitig verwendbares Unterrichtsmedium anbot. Solche Schriften konnten sowohl von Lehrern als didaktische Hilfsmittel genutzt, als auch im häuslichen Unterricht oder zum Selbststudium herangezogen werden. Velten zeigt den innovativen Ansatz von Ickelsamers Leselernmethode, die ihren Ausgang nicht 
mehr von den Buchstaben, sondern von den Lauten nahm und zunächst auf eine Bewusstheit der Lernenden über die phonetische Artikulation abzielte. Auch kritisierte Ickelsamer den bis dahin üblichen Rückgriff auf die lateinische Grammatik beim deutschen Schriftspracherwerb und erarbeitete eine alternative deutsche Sprachlehre. Dass sowohl die Leselernmethode als auch die Anleitung zum Verständnis der grammatischen Struktur der Sprache in ein umfassenderes religiöses Bildungskonzept eingebettet waren, macht Velten überzeugend deutlich.

Schulisches Lesen und Lernen waren in der frühen Neuzeit oft eng mit religiösen Erziehungsabsichten verknüpft. Dies gilt in besonderem Maße für den Elementarunterricht, während sich auf den weiterführenden Stufen immer auch die fachlichen Ausbildungsansprüche geltend machten, die die religiösen Intentionen relativieren konnten (vgl. die verschiedenen Beiträge in Musolff et al. 2008). Die Verwobenheit von Religion und Erziehung zeigen die Beiträge von Stefan Ehrenpreis und John Exalto an Beispielen aus Franken und den Niederlanden im 16. und 17. Jh. auf. Ehrenpreis beobachtet Prozesse des Lesens und Lernens an den Praktiken der Katechese. Je nach Blickwinkel stellt sich diese teils als eine Form der Sozialdisziplinierung, teils als ein Medium zum Erlernen der Kulturtechniken dar. Am Beispiel der Reichsstadt Nürnberg und des Fürstentums Ansbach zeichnet Ehrenpreis in seinem Beitrag Katechismen und Katechese. Frühneuzeitliche Schulbücher als politisch-sozialer Konfliktstoff im Konfessionellen Zeitalter nach, inwiefern die Katechese im 16. und 17. Jh. eine Schnittstelle der erzieherischen Ansprüche von (Elementar-)Schule, Kirchengemeinde und Familie bildete. So zeigten etwa die öffentlichen Katechismusprüfungen sonntags nach dem Gottesdienst, was die Kinder im schulischen Unterricht gelernt hatten. Dies wiederum warf ein Licht auf den Grad der Integration einer Familie in das bestehende Normengefüge der Gemeinde. Das Interesse der Obrigkeiten an der Katechese erhellt auch aus der Privilegierung regionaler Buchdrucker, der Kontrolle des Buchmarkts und der Katechismusproduktion sowie der Buchzensur.

Das Erlernen des Lesens und Schreibens in den drei ersten Schuljahren erläutert John Exalto mit Blick auf Alphabet, Bibel, Katechismus: Das ABC der vormodernen Grundschule in den Niederlanden. Auffallend früh gingen die Kinder zur Schule, nämlich im Alter von vier oder fünf Jahren. Welche Unterrichtsmaterialien für das Lesenlernen im Religionsunterricht und für das fortgeschrittenere Lesen erwarteten diese Kinder? Exalto zeigt u.a. die Verwendung des unter Leitung des polnischen Theologen Jan Łaski verfassten „Hahnenbuchs“ sowie der Kindertestamentesammlung des reformierten Pfarrers Wilhelmus Eversdijk im Unterricht auf. Testamente bildeten auch eine verbreitete außerschulische Lektüre. Darüber hinaus macht Exalto deutlich, inwiefern im niederländischen Elementarschulwesen (und nicht nur dort) vielfältige Impulse zur Ausbildung des eigenen religiösen und kulturellen Selbstverständnisses genutzt wurden. Dass dabei viel auswendig gelernt wurde, betrachteten die Zeitgenossen insgesamt als selbstverständlichen Bestandteil der Aneignung geistiger Gehalte.

Zum Elementarunterricht gehörte seit der frühen Neuzeit das Rechnen. Das ist nicht selbstverständlich: Rechenunterricht hat in den offiziellen Lehrplänen und Schulordnungen der weiterführenden Schulen bis in das 18. Jh. hinein oft nur eine marginale Rolle gespielt, auch wenn dies nicht bedeutet, dass er keinen Ort im Curriculum gehabt hätte (vgl. Bruchhäuser 2005, S. 105; Musolff 2005, S. 194 f.). Daran, dass die Schulordnungen 
für den Elementarunterricht das Rechnenlernen demgegenüber häufig ausdrücklich vorsahen, erinnert Kurt Wesoly in seinem Beitrag über Rechenunterricht und Rechenbücher bis zur Mitte des 18. Jahrhunderts, mit besonderer Berücksichtigung des Herzogtums Berg. Schwerpunkte und Niveau dieses Rechenunterrichts, so Wesolys Prämisse, werden u.a. an den überlieferten Lehrwerken fassbar. Er stellt fest, dass trotz z. T. anderslautender Vorreden in den Lehrwerken deren Adressaten oft weniger die Kinder als die Rechenmeister gewesen sind, die mit diesen Büchern unterrichteten. Abnehmer dieser Lehrwerke waren - wie im Fall Ickelsamers - auch Autodidakten. Wesoly zeigt, an welch praxisnahen Beispielen vornehmlich aus der kaufmännischen Tätigkeit das Rechenbuch von Servatius Schlyper aus dem Jahr 1734 veranschaulichte, was Kinder und Jugendliche zu lernen hatten, die oder deren Eltern für sie den Kaufmannsberuf anstrebten. Dazu gehörten das Rechnen mit Brüchen, Umrechnungen von Maßen, Gewichten und Münzen und v. a. die Regula de Tri, der Dreisatz. Dass auch die handwerklichen und bäuerlichen Schichten einschlägige Kenntnisse gehabt haben mussten, macht Wesoly mit Verweis auf Rechnungslegungen, Geschäftsbücher und Steuerberechnungen deutlich.

Fragen nach dem Absatz von Schulbüchern und Lehrmaterial für den Primarunterricht stehen im Mittelpunkt des bereits erwähnten wirtschaftsgeschichtlich angelegten Beitrags von Emmanuelle Chapron über Das Elementarschulbuch im 18. Jahrhundert: Räumliche Ausbreitung und Handelspraktiken zwischen Paris und der Champagne (1680-1730). Gestützt auf eine außergewöhnlich gute Überlieferung des Verwaltungsschrifttums erschließt Chapron die Beziehungen zwischen Druckereien und Buchhandel und veranschaulicht die Verbreitung von Lehrwerken mit Blick auf die lokalen und überregionalen Marktstrukturen. Im Zusammenhang dieser Verbreitung spielte die Erteilung von Druckprivilegien eine wichtige Rolle. Am Beispiel der Ursulinen wird der Erwerb von Schulbüchern im Klassensatz nachgewiesen.

Während die Lehrwerke für den Elementarunterricht zumeist explizit für den Unterricht verfasst und in den Offizinen produziert wurden, lagen dem Sekundarunterricht auch Werke zugrunde, die von ihren Autoren keineswegs als Unterrichtsliteratur gedacht waren. Solche Werke, wie z. B. die Rhetorik des Aristoteles oder Briefe Ciceros, sind oft in einer für den Schulgebrauch eingerichteten Form erschienen. In seinem Beitrag Schulbücher und Lektüren in der Unterrichtspraxis an böhmischen und mährischen Lateinschulen des 16. und frühen 17. Jahrhunderts weist Martin Holý die zahlreichen für den Unterrichtsgebrauch an städtischen, kirchlichen oder privaten Lateinschulen bestimmten Texte und Bücher nach. Dabei stehen die primär schulisch intendierten Werke im Vordergrund, insbesondere solche Lehrbücher und andere Texte, die für das Lateinlernen und damit für das Hauptfach dieser Schulen genutzt wurden: Grammatiken, verschiedene Wörterbücher, Werke zur Rhetorik usw.

Jean-Luc Le Cam stellt Schulbücher zwischen Vorschrift, Angebot und Gebrauch am Beispiel des braunschweigischen Gelehrtenschulwesens im 17. Jahrhundert vor. Als sehr ergiebige Quellen haben sich die schon genannten, von ihm ausgewerteten Inspektionsberichte Christoph Schraders erwiesen. Sie erlauben einen Einblick in die Schulpolitik Herzog Augusts des Jüngeren, der genaue Vorschriften zu den zu nutzenden Schulbüchern erließ. Damit begegnete die Obrigkeit u. a. dem zu häufigen Austausch von Schulbüchern bzw. ihrem parallelen Gebrauch, was zumeist dem Lehrerwechsel geschuldet war. In jährlichen Visitationen wurde überprüft, ob die tatsächlich genutzten Schulbücher 
mit denen übereinstimmten, die in der Schulordnung von 1651 vorgesehen waren. Die strikten Vorgaben begünstigten die Hofdruckerei der Gebrüder Stern, denn es sollten vorzugsweise solche Lehrbücher verwendet werden, die im Land gedruckt wurden. Trotz der obrigkeitlichen Normierung und Kontrolle der Schulbücher und trotz der merkantilistischen Politik konstatiert der Beitrag eine fortwährende Diskrepanz zwischen Norm und Praxis, wobei dieser Befund unterschiedlich ausfällt, je nachdem, ob Lateinschulen mit nur wenigen Klassenstufen oder Gymnasien inspiziert wurden. Auch spielte die Entfernung einer Schule zur Residenz eine nicht unerhebliche Rolle für die Durchsetzung der herzoglichen Vorgaben. Dass die Schulreform in Braunschweig-Wolfenbüttel keineswegs originell war, sondern sich am Vorbild Leidens orientierte, beweist einmal mehr den enormen kulturellen Einfluss der Niederlande auf das Bildungswesen in den deutschen Ländern.

Den Unterricht der Mädchen bringt Anne Conrad in ihrem Beitrag über Lernmaterialien und Lesepraxis an Ursulinenschulen des 17. und 18. Jahrhunderts in den Blick. Sie stützt sich auf die nachweisbaren Buchbestände, auf die Lehrende und Lernende in ihrem Unterricht zurückgreifen konnten. Indem sie die allgemeinen Angaben des Pariser Schulreglements von 1652 zu Schulorganisation und Erziehungszielen sowie zur religiösen Bildung in den Ursulinenkonventen mit dem rekonstruierbaren Bibliotheksbestand des Erfurter Ursulinenklosters in Beziehung setzt, gewinnt sie eine Vorstellung von der inhaltlich-curricularen Ausgestaltung des Unterrichts. Wie bei einer geistlichen Gemeinschaft, die sich zunächst der Katechese, dann verstärkt der Mädchenbildung gewidmet hat, zu erwarten ist, weist der Bibliotheksbestand eine Reihe von Katechismusausgaben und anderen religiösen Schriften auf. Aber es finden sich auch "gelehrte Lektüren" weltlichen Inhalts, z. B. eine griechisch-lateinische Aristoteles-Ausgabe oder Titel zur Geographie und Zeitgeschichte. Auch im Vergleich mit dem Konvent der Würzburger Ursulinen bleibt die Schwierigkeit, aus dem Nachweis vorhandener Bücher auf die wirklichen Lektürepraktiken zu schließen. Die Problematik der Quellenlage verdeutlicht einmal mehr, dass auch normative Quellen wie das zitierte Schulreglement nicht außer Acht gelassen werden dürfen, sondern dass sie - im Abgleich mit Quellen, die näher an die Lesepraxis heranführen - durchaus Hinweise auf diese Praxis geben können.

Inwiefern Gebrauchsspuren der von Schülern und Lehrern genutzten Lehrwerke in Kombination mit anderen Quellen, insbesondere Disputationen und einem Lektionsplan, über die curricularen Schwerpunkte und die methodische Gestaltung des Unterrichts Aufschluss geben können, veranschaulicht der Beitrag von Stephanie Hellekamps und HansUlrich Musolff Zur Didaktik der Frühaufklärung. Schulbücher und Lektüren für den Philosophieunterricht am Soester Gymnasium zu Beginn des 18. Jahrhunderts. Die Glossierung, die ein Schüler an dem Lehrbuch seines Lehrers, des Soester Rektors Rumpaeus, anbrachte, deutet auf einen inhaltlich und methodisch orthodoxen Unterricht hin. Demgegenüber lassen die Glossen des Konrektors Marci, die sich in dem von ihm benutzten Exemplar von Descartes' Passiones animae finden, auf ein anderes didaktisches Konzept schließen. Sein eher diskursiv angelegter Unterricht zielte auf das kritische Selbstdenken der Schüler. Wie groß der tatsächliche Lernerfolg der gemäß einer cartesianisch inspirierten Didaktik unterrichteten Schüler war, lässt sich auf der Grundlage der vorliegenden Quellen allerdings nicht mit Sicherheit feststellen. Wohl aber lässt sich die Häufigkeits- 
verteilung von eher orthodox oder eher diskursiv ausgerichtetem Unterricht für den Zeitraum 1600-1750 auch quantitativ bestimmen.

Gegenüber dem frühneuzeitlichen Lernen an städtischen Lateinschulen und Gymnasien sowie an den Schulen der Konvente deuten eine hochgradig ausgewählte Lehrerschaft und die Repräsentativität und Vielfalt der zur Verfügung stehenden Lehrbücher und Lernmaterialien auf das besondere Anspruchsniveau der Adelserziehung hin. Welche Lernerfolge unter diesen Bedingungen möglich waren, veranschaulicht der Beitrag von Sylvène Edouard über The Books used by Mary Stuart for the exercise on „Acquérir dela doctrine" (1554-1555). Edouard untersucht die verschriftlichten Ergebnisse tatsächlicher Lektüren an einer besonders aussagekräftigen Quelle, nämlich einem Korpus von 64 in Briefform gehaltenen Texten, die die junge Königin von Schottland im Alter von elf Jahren auf Anregung ihres Lehrers verfasst hat. Diese Texte sind im Kontext rhetorischer Übungen entstanden, denen eine Reihe von Werken zugrundelag, die als vorbildlich anerkannt waren. Der Beitrag zeigt, auf welche Werke sich Maria Stuart gestützt hat (u. a. auf Texte von Erasmus und Plutarch sowie eine Fabel von Äsop), und wie sie sich mit ihren Vorlagen auseinandergesetzt hat. Die Aneignung der Texte durch die junge Rezipientin erfolgte durch nachahmendes Schreiben, das die Intensität der wiederholten Lektüren anschaulich macht.

Die mehrfach erwähnte Diskrepanz zwischen Norm und Praxis, die die Historikerin zur Nutzung verschiedenartiger Quellen nötigt, wird auch an dem Beitrag von Dominique Picco über Bücher und Lektüren für junge Mädchen. Das Beispiel Saint-Cyr (Ende des 17. Jahrhunderts bis 1750) fassbar. Dass die „gelehrte Frau“ dem zeitgenössischen Rollenbild in der Erziehung der jungen Mädchen keineswegs entsprach, veranschaulicht Picco insbesondere an der Korrespondenz der Madame de Maintenon sowie an verschiedenen Schriften zur weiblichen Erziehung Claude Fleurys und François de Fénelons. Die erzieherische Wirklichkeit entsprach den zeitgenössischen pädagogischen Theorien aber nur zum Teil. Dies zeigt das Beispiel der von Ludwig XIV. auf Betreiben Madame de Maintenons gegründeten Erziehungsanstalt von Saint-Cyr, die für die Töchter verarmter Adeliger eingerichtet wurde. Während die pädagogische Konzeption auf eine im Wesentlichen religiöse Erziehung abzielte und entsprechende Lektüren vorsah, zeigen die Inventarverzeichnisse der Bibliothek darüber hinaus auch wissenschaftliche Werke, Bücher zur Geschichte und zu den Künsten.

Den Bildungsgang zweier französischer Prinzen erläutert Pascale Mormiche. In ihrem Beitrag Lehrbücher und Lektüren zur Erziehung französischer Prinzen im 17. und 18. Jahrhundert untersucht sie die Bibliothek mit ihren je verschiedenen Beständen, die der junge König Ludwig XV. vor und um 1730 sowie ab ca. 1736 sein Sohn, der Dauphin, zu Unterrichtszwecken nutzten. Diese Bibliothek repräsentiert das Niveau der fortgeschrittenen Bildung, da die elementare Bildung im Kreis der Gouvernanten stattfand. Mormiche rekonstruiert anhand des überlieferten Bibliothekskataloges aus dem Jahr 1730, welchen Bildungshorizont die Bibliothek für den jungen Ludwig XV. repräsentiert haben muss. Mit Blick auf die inhaltliche Vielschichtigkeit des Bestandes weist sie frühere Forschungsmeinungen zurück, denen zufolge Erziehung und Unterricht Ludwigs $\mathrm{XV}$. eindimensional gewesen seien. Im Vergleich zu dessen Bildungsgang zeuge der des Dauphin - wiederum ausweislich des für seine Unterrichtszeit rekonstruierbaren Bibliotheksbestandes - von einer stärkeren Anlehnung an die Curricula der Collèges. Unter der 
Prämisse, dass die Prinzen die ihnen verfügbaren Bücher auch wirklich gelesen haben, sei ihr Bildungsniveau nach dem Ende ihrer Unterrichtszeit, so führt Mormiche aus, beachtlich gewesen.

Dem Use of the French „Abrégés d'histoire“ during the 17th and 18th Centuries, die in manchen Collèges sowie für die Prinzenerziehung verwendet wurden, ist der Beitrag von Annie Bruter gewidmet. Diese historischen Tabellen mit Namenlisten von Herrschern sollten den Schülern ein chronologisches Gerüst vermitteln. Aus zeitgenössischen Methodenbüchern sowie der Einrichtung dieser Tabellenwerke schließt Bruter auf den Gebrauch durch die Schüler: Namenlisten in Versform wurden memoriert und um weitere Fakten ergänzt. Es ging darum, die Sukzessionen von Fürsten und Königen sowie Ereignisabfolgen in einer Zeit lehrbar und lernbar zu machen, als Geschichte noch kein eigentliches Fach des Lehrplans war und historische Studien in der Lektüre der antiken Historiographen bestanden.

Die in diesem Band versammelten Beiträge verdeutlichen die v.a. methodischen Schwierigkeiten, auf die Ebene des tatsächlichen Unterrichts in der Vormoderne vorzudringen. Nicht in jeder Untersuchung kann der Nachweis der wirklichen oder zumindest wahrscheinlichen Lektüren erbracht werden. Mitunter wird mit Indizien für die allenfalls mögliche Nutzung von Lehrwerken argumentiert. Dies betrifft insbesondere die Beiträge, die bisher nur die in einem Untersuchungszeitraum und Untersuchungsgebiet im Allgemeinen vorfindlichen Buchbestände auflisten können. Bezogen auf einzelne Regionen sind aber auch präzisere Aussagen zur Nutzung, Verbreitung und Normierung von Schulbüchern möglich. Es gab Märkte der Schulbuch- und Unterrichtsliteratur, wie die lokale Studie zur Champagne zeigt, denn Unterricht fand weitgehend auf schriftgestützter Basis statt. Dies gilt schon für die Zeit vor dem Buchdruck, worauf die Schul-Handschriften zu den Fabulae Avians und den (deutschen) Disticha Catonis hinweisen. Die Auswertung überlieferter Bibliotheks- und Inventarverzeichnisse verdeutlicht den Anspruch des zu Lernenden vom Erwerb der ersten Fremdsprache, des Lateinischen, an bis zur Lektüre antiker Autoren in der Originalsprache und zeitgenössischer philosophischer, historischer und geographischer Darstellungen und Überblicke. Als durchgängige Schwierigkeit erweist es sich, Aussagen über die konkreten Vermittlungsformen im Unterricht zu machen. Um das nur schwer erforschbare Verhältnis von Mündlichkeit und Schriftlichkeit im Unterricht weiter aufzuhellen oder das Maß selbständigen Lernens festzustellen, ist der Rückgriff auf die authentischen Handschriften und Bücher sowie auf Besitzvermerke und Glossen unerlässlich. Eine Neubewertung erfährt schließlich das Auswendiglernen: Das Memorieren diente der Versenkung in einen Inhalt und dessen Aneignung, so dass er als geistiges Potential fortwirken konnte.

Dass die 13. AVE-Tagung stattfinden konnte und deren Ergebnisse nun in diesem Band vorgestellt werden können, ist dem Engagement vieler einzelner Personen und der Großzügigkeit mehrerer Institutionen zu verdanken. Ohne die beträchtliche finanzielle Förderung durch das $\mathrm{ZiF}$ in Bielefeld wäre es nicht möglich gewesen, dieses interdisziplinäre bildungshistorische Gespräch durchzuführen. Die unbürokratisch-rasche Zusage finanzieller Unterstützung durch das Institut français d'histoire en Allemagne hat es erlaubt, dass wir Simultanübersetzungen vom Französischen ins Deutsche und umgekehrt anbieten konnten. Die Université de Bretagne occidentale hat die Übersetzung einiger Texte für die Publikation dieses Bandes gefördert. Allen drei Institutionen sei an dieser Stelle 
ganz herzlich für ihre Unterstützung gedankt. Wir danken den Simultandolmetscherinnen Steffi Gleißner und Heide Ruppert, die viel zur Lebendigkeit des Austausches beigetragen haben, und Dietmar Trempenau für seine sehr sorgfältigen Übersetzungen einiger französischer Beiträge ins Deutsche. Ob eine Tagung gelingt, hat immer auch viel mit der Atmosphäre des Ortes zu tun, an der sie stattfindet. Wir sind Trixi Valentin und dem Team vom $\mathrm{ZiF}$ für das sehr angenehme Arbeitsklima und die ausgezeichnete Organisation der Tagung zu großem Dank verpflichtet. $\mathrm{Zu}$ danken haben wir ferner den Moderatorinnen und Moderatoren der einzelnen Sektionen für ihre sachkundige Diskussionsleitung: Alwin Hanschmidt (Vechta), Jürgen Helmchen (Münster), Juliane Jacobi (Potsdam), Hans-Ulrich Musolff (Münster), Andreas Rutz (Bonn) und Serge Tomamichel (Lyon). Jürgen Helmchen danken wir auch für seine simultane Übersetzung einiger deutscher Vorträge ins Französische, Juliane Jacobi danken wir für ihre Unterstützung bei der Bearbeitung der Übersetzungen, und Andrea Hofmeister (Göttingen) und nochmals Andreas Rutz für die Erstellung der Tagungsberichte. Nicht zuletzt gebührt unser Dank allen Referentinnen und Referenten und allen Teilnehmern und Teilnehmerinnen für ihr Bemühen um die gemeinsame Sache.

\section{Literatur}

Albertini, P. (1986). L'Enseignement classique à travers les exercises manuscrits des élèves. INRP, Collection Rapports de recherche, 5.

Baldzuhn, M. (2009). Schulbücher im Trivium des Mittelalters und der Frühen Neuzeit. Die Verschriftlichung von Unterricht in der Text- und Überlieferungsgeschichte der ,Fabulae “ Avians und der deutschen „Disticha Catonis “(Bd. 2). Berlin: de Gruyter. (=Quellen und Forschungen zur Literatur- und Kulturgeschichte; 44).

Bernhard, M. (1996). Boethius im mittelalterlichen Schulunterricht. In M. Kintzinger u. a. (Hrsg.), Schule und Schüler im Mittelalter. Beiträge zur europäischen Bildungsgeschichte des 9. bis 15. Jahrhunderts (S. 11-27). Köln u. a.: Böhlau.

Blair, A. (2008). Student manuscripts and the textbook. In E. Campi u. a. (Hrsg.), Scholarly Knowledge. Textbooks in early modern Europe (S.39-73). Genf: Droz. (=Travaux d'Humanisme et Renaissance; CDXLVII).

Bodemann, U., \& Dabrowski, C. (2000). Handschriften der Ulmer Lateinschule. Überlieferungsbefund und Interpretationsansätze. In K. Grubmüller (Hrsg.), Schulliteratur im späten Mittelalter (S. 11-47). München: Fink.

Bodemann, U., \& Grubmüller, K. (1992). Schriftliche Anleitung zu mündlicher Kommunikation: Die Schülergesprächsbüchlein des späten Mittelalters. In H. Keller u. a. (Hrsg.), Pragmatische Schriftlichkeit im Mittelalter. Erscheinungsformen und Entwicklungsstufen. Akten des Internationalen Kolloquiums 17.-19. Mai 1989 (S. 177-193). München: Fink.

Bruchhäuser, H.-P. (2005). Die Berufsbildung deutscher Kaufleute bis zur Mitte des 16. Jahrhunderts. In A. Hanschmidt \& H.-U. Musolff (Hrsg.), Elementarbildung und Berufsausbildung 1450-1750 (S. 95-107). Köln u. a.: Böhlau.

Caspard, P. (Hrsg.). (1990). Travaux d'élèves. Pour une histoire des performances scolaires et de leur évaluation, 1720-1830. Histoire de l'éducation, 46(Spécial), 27-49.

Compère, M.-M., \& Pralon-Julia, D. (1992). Performances scolaires de collégiens sous l'Ancien Régime. Études d'exercices latins rédigés au collège Louis-le-Grand vers 1720. Paris: Publications de la Sorbonne. 
Dainville, O. de (1947/1978). Librairies d'écoliers toulousains à la fin du seizième siècle. In M.-M. Compère (Hrsg.), L'éducation des jésuites (XVIe-XVIIIe siècles) (S.267-278). Paris: de Minuit.

Desgraves, L. (1973). L'inventaire du fonds de livres du libraire bordelais Jacques Mongiron-Millanges en 1672. Revue française d'histoire du livre, Nouv. Série, 3, 125-171.

Grubmüller, K. (Hrsg.). (2000). Schulliteratur im späten Mittelalter. München: Fink.

Henkel, N. (1988). Deutsche Übersetzungen lateinischer Schultexte. Ihre Verbreitung und Funktion im Mittelalter und in der frühen Neuzeit. Mit einem Verzeichnis der Texte. München u. a.: Artemis. (=Münchener Texte und Untersuchungen zur deutschen Literatur des Mittelalters; 90).

Hergenhahn, R. (2007). Brevis Arithmetices Introdvctio 1549. Einblick in den gymnasialen Rechenunterricht. Beiträge zur Geschichte Dortmunds und der Grafschaft Mark, 96/97, 77-121.

Jacobi, J. (2008). Zwischen „,nöthigen Wissenschaften“ und ,gottesfurcht“. Protestantische Mädchenschulen von der Reformation bis zum 18. Jahrhundert. In H.-U. Musolff, J. Jacobi, \& J.-L. Le Cam (Hrsg.), Säkularisierung vor der Aufklärung? Bildung, Kirche und Religion 1500 1750 (S. 253-274). Köln u. a.: Böhlau.

Klieme, E., et al. (Hrsg.). (2010). PISA 2009. Bilanz nach einem Jahrzehnt. Münster u. a.: Waxmann.

Martin, H.-J. (2000). La naissance du livre moderne (XIVe-XVIIe siècles). Tours: Èditions du Cercle de la Liberairie (Avec la collaboration de J.-M. Chatelain, I. Diu, A. Le Dividich \& L. Pinon).

Musolff, H.-U., Jacobi, J., \& Le Cam, J.-L. (Hrsg.). (2008). Säkularisierung vor der Aufklärung? Bildung, Kirche und Religion 1500-1750. Köln u. a.: Böhlau.

Musolff, H.-U. (2005). Das Soester Schulwesen und seine Ausbildungsfunktion für nicht-akademische Berufe. In A. Hanschmidt \& H.-U. Musolff (Hrsg.), Elementarbildung und Berufsausbildung 1450-1750 (S. 167-205). Köln u. a.: Böhlau.

Volpilhac-Auger, C. (Hrsg.). (2000). La collection Ad usum Delphini. L'Antiquité au miroir du Grand Siècle. Genoble: Ellug. 